\title{
Phase shifts and in-medium cross sections for dressed nucleons in nuclear matter
}

\author{
W. H. Dickhoff, C. C. Gearhart, * and E. P. Roth \\ Department of Physics, Washington University, St. Louis, Missouri 63130
}

A. Polls and A. Ramos

Departament d'Estructura de la Matèria, Facultat de Física, Diagonal 647, E-08028 Barcelona, Spain

(Received 29 July 1999; published 16 November 1999)

\begin{abstract}
The dressing of nucleons as embodied in single-particle spectral functions is incorporated in the description of nucleon-nucleon scattering in nuclear matter at a density corresponding to $k_{F}=1.36 \mathrm{fm}^{-1}$. In order to clarify the new features associated with the complete off-shell behavior of the single-particle motion, results involving mean-field particles are also presented with special emphasis on the behavior of the phase shifts when bound pair states occur. Both the ${ }^{1} S_{0}$ and ${ }^{3} S_{1-}{ }^{3} D_{1}$ channels exhibit this feature at the considered density for mean-field particles at zero temperature. An important tool to assess the effect of the dressing of the particles is the two-particle density of states. A sizable reduction with respect to the mean-field density of states is obtained. At $2 \epsilon_{F}$ this reduction corresponds to $z_{k_{F}}^{2}$, where $z_{k_{F}}$ is the strength of the quasiparticle pole at $k_{F}$, and it can therefore be as large as 0.5 . This reduction has significant consequences for the strength of pairing correlations both in the ${ }^{3} S_{1-}{ }^{3} D_{1}$ channel where it leads to a dramatic decrease of the attraction at the Fermi energy and for the ${ }^{1} S_{0}$ channel which no longer shows a pairing signal. Phase shifts and cross sections for dressed particles are determined based on expressions which fold the effective interaction with the dressed but noninteracting two-particle spectral function. This folding procedure yields similar results to an "on-shell", prescription reminiscent of the result for free or mean-field particles, except for cross sections deep in the Fermi sea. Comparison of phase shifts and cross sections to the case of mean-field particles indicates that smaller phase shifts in an absolute sense and considerable reductions of the in-medium cross sections for dressed particles are obtained. It is shown that while in many cases these results imply a weakening of the effective interaction, this is not the case for ${ }^{1} S_{0}$ interactions deep in the Fermi sea. [S0556-2813(99)06612-1]
\end{abstract}

PACS number(s): 21.65.+f, 21.30.Fe

\section{INTRODUCTION}

For various reasons the study of the interaction between nucleons in the nuclear medium has retained a considerable amount of interest over the years. The construction of the effective interaction to be used in shell-model calculations is but one example $[1,2]$. The modification of the effective interaction in nuclear or neutron matter with increasing density remains a challenging issue for a deeper understanding of these systems [3]. Recent interest in the study of the interaction between nucleons in the nuclear medium has been generated by the experimental developments involving the $\left(e, e^{\prime} p p\right)$ reaction $[4,5]$. The potential selectivity of this reaction for the removal of ${ }^{1} S_{0}$ proton pairs to certain final states and the absence of large contributions from two-body currents to these transitions may allow the study of the interaction between protons in the medium at short relative distances [6].

Of particular interest is the exploration of the "inmedium" interaction in the context of transport-theory descriptions of heavy-ion reactions [7]. Typical analyses simulate the dynamics of a heavy-ion reaction on the basis of kinetic equations like the Boltzmann-Uehling-Uhlenbeck (BUU) equation [8]. An essential ingredient in these BUU

\footnotetext{
*Present address: Ford Scientific Research Laboratory, 20000 Rotunda Drive, P.O. Box 2053, MD 2115, Rm 2115, SRL, Dearborn, MI 48121-2053.
}

calculations is the nucleon-nucleon cross section in the medium. Attempts to include medium-modified cross sections in such calculations have been described in Ref. [9]. Calculations of the cross section between nucleons in nuclear matter have been reported in Refs. [10-19]. Some recent issues that have emerged from this work include the enhancement of the cross section at finite temperature due to the vicinity of a pairing instability [16], the sensitivity of the cross section to the choice of the single-particle (sp) spectrum at zero temperature [17], the density and energy dependence [18], and temperature dependence of the cross sections [19].

All results obtained in Refs. [10-19] have been generated under the assumption that the sp motion of the nucleon in the medium is that of a mean-field ( $\mathrm{mf}$ ) particle. Under this assumption the scattering process in the medium takes place between nucleons which at most have a sp spectrum different from free space but are otherwise unaffected by the presence of other nucleons except for the Pauli principle related to a $\mathrm{mf}$ Fermi gas. This assumption has been contradicted unequivocally in recent years for finite nuclei by a careful analysis of the $\left(e, e^{\prime} p\right)$ reaction. This analysis demonstrates that the sp strength is not completely concentrated at one sp energy but for states accessed in this reaction has a distribution characterized by about $2 / 3$ of the strength close to or spread around the expected sp energy [20]. Some additional strength is inferred [21,22] at higher missing energy, leading to occupation numbers of about $75 \%$ close to the Fermi energy in ${ }^{208} \mathrm{~Pb}$. Many-body calculations of the spectral function of nuclear matter using realistic interactions $[23,24]$ gen- 
erate a similar picture at normal nuclear matter density. It is therefore fair to say that both on the basis of experimental results as well as theoretical calculations for nuclear matter, it is prudent to consider the sp dressing of the nucleons in matter and the effect this has on the scattering process.

Preliminary results related to the present work have been presented in Refs. [25-28]. In a recent paper [29] one of the present authors has attempted to provide a framework to interpret the results obtained in a ladder-diagram calculation of the two-body propagator which employs fully dressed $\mathrm{sp}$ propagators. One of the consequences of employing dressed particles is the localization of the two-body propagator in coordinate space, severely limiting the range of the propagation for most energies. This feature is due to the presence, at a given energy, of a range of momenta which determine the relative wave function of the propagating particles. While for $\mathrm{mf}$ particles a unique (on-shell) momentum characterizes the relative wave function which therefore corresponds to a plane wave (or spherical wave), the presence of different momentum components implies that the determination of phase shifts and cross sections requires some kind of folding procedure over these momenta in the case of dressed particles. In Ref. [29] a set of expressions has been introduced to characterize the scattering event of dressed particles involving such a folding procedure. Results for phase shifts and cross sections obtained from these expressions are compared in this paper with the corresponding results propagating free and $\mathrm{mf}$ particles using the Reid soft-core potential [30].

In Sec. II A the relation between the two-body propagator and the effective interaction is summarized for free, $\mathrm{mf}$, and dressed particles. In Sec. II B the link between the description of the scattering process of free particles and the twobody propagator is presented including the case of coupled channels as required for the nuclear tensor force. The slight modifications required for the description of the scattering of $\mathrm{mf}$ particles are discussed in Sec. IIC. Useful material describing the relation between phase shifts at energy thresholds and bound states through a generalization of Levinson's theorem can be found in Ref. [31]. Relevant results required for the description of the scattering of dressed particles are collected in Sec. II D. This subsection also includes a discussion of the two-body spectral density and two-body spectral function for noninteracting but dressed particles. The latter quantity features prominently in determining the two-body density of states which is compared with the corresponding results for free and $\mathrm{mf}$ particles at zero total momentum. A brief discussion of the method of calculating the two-body propagator in a partial-wave momentum space representation is presented. Expressions used for calculating the phase shifts and cross sections of dressed particles based on the work of Ref. [29] are also collected in Sec. II D.

Results for phase shifts involving the propagation of $\mathrm{mf}$ particles are discussed in Sec. III. This section is intended on the one hand to make contact with Ref. [31] where the scattering of ${ }^{3} \mathrm{He}$ atoms was studied based on the use of a very strongly repulsive central interaction, while on the other hand clarifying, in the nuclear case, the relation between phase shifts and the possible occurrence of bound pair states.
These results also provide the background for the presentation of phase shifts and cross sections for the propagation of dressed particles which is given in Sec. IV. A summary and conclusions are presented in Sec. V.

\section{SCATTERING FORMALISM EMPLOYING THE TWO-BODY PROPAGATOR}

The purpose of the present section is to gather all results relevant for the calculation of phase shifts between nucleons in the nuclear medium. The physics included in the description of the scattering in the medium involves the proper inclusion of short-range correlations by means of summing all ladder diagrams for a realistic nucleon-nucleon interaction. The corresponding ladder-summed two-body effective interaction can then be linked to the two-body propagator which provides a convenient tool to describe the scattering in the medium [29].

\section{A. Two-body propagator and effective interaction}

For the purpose of the present work it is sufficient to consider the two-time two-particle propagator

$$
\begin{aligned}
& g^{I I}\left(\boldsymbol{k}_{1} \boldsymbol{k}_{2} ; \boldsymbol{k}_{3} \boldsymbol{k}_{4} ; t_{1}-t_{2}\right) \\
& \quad=-i\left\langle\Psi_{0}^{A}\left|T\left\{a_{\boldsymbol{k}_{2}}\left(t_{1}\right) a_{\boldsymbol{k}_{1}}\left(t_{1}\right) a_{\boldsymbol{k}_{3}}^{\dagger}\left(t_{2}\right) a_{\boldsymbol{k}_{4}}^{\dagger}\left(t_{2}\right)\right\}\right| \Psi_{0}^{A}\right\rangle,
\end{aligned}
$$

given here in the momentum representation, while spin and isospin indices are suppressed. This propagator depends on the conserved total momentum $\boldsymbol{K}=\boldsymbol{k}_{1}+\boldsymbol{k}_{2}=\boldsymbol{k}_{3}+\boldsymbol{k}_{4}$ in the medium. To facilitate a comparison with the scattering of particles in free space only results for the case $|\boldsymbol{K}|=0$ will be considered in Secs. III and IV. Extension of the present work to deal with the case of nonzero total momentum requires a numerically reliable calculation of the noninteracting propagator of dressed particles in the medium and will be presented elsewhere [33]. The remaining momentum dependence of the propagator can now be associated with the relative momentum of the pair of removal operators in Eq. (1), given by $\boldsymbol{k}=\frac{1}{2}\left(\boldsymbol{k}_{1}-\boldsymbol{k}_{2}\right)$, and of the pair of addition operators given by $\boldsymbol{k}^{\prime}=\frac{1}{2}\left(\boldsymbol{k}_{3}-\boldsymbol{k}_{4}\right)$. Since only ladder diagrams are considered together with a static bare $N N$ interaction, the corresponding integral equation for the propagator can be written as

$$
\begin{aligned}
g^{I I}\left(\boldsymbol{k}, \boldsymbol{k}^{\prime} ; \boldsymbol{K}, \Omega\right)= & g_{f}^{I I}\left(\boldsymbol{k}, \boldsymbol{k}^{\prime} ; \boldsymbol{K}, \Omega\right) \\
& +g_{f}^{I I}(\boldsymbol{k} ; \boldsymbol{K}, \Omega) \int d^{3} q\langle\boldsymbol{k}|V| \boldsymbol{q}\rangle g^{I I}\left(\boldsymbol{q}, \boldsymbol{k}^{\prime} ; \boldsymbol{K}, \Omega\right) \\
= & g_{f}^{I I}\left(\boldsymbol{k}, \boldsymbol{k}^{\prime} ; \boldsymbol{K}, \Omega\right)+g_{f}^{I I}(\boldsymbol{k} ; \boldsymbol{K}, \Omega) \\
& \times\left\langle\boldsymbol{k}|\Gamma(\boldsymbol{K}, \Omega)| \boldsymbol{k}^{\prime}\right\rangle g_{f}^{I I}\left(\boldsymbol{k}^{\prime} ; \boldsymbol{K}, \Omega\right)
\end{aligned}
$$

where

$$
g_{f}^{I I}\left(\boldsymbol{k}, \boldsymbol{k}^{\prime} ; \boldsymbol{K}, \Omega\right)=\delta\left(\boldsymbol{k}-\boldsymbol{k}^{\prime}\right) g_{f}^{I I}(\boldsymbol{k} ; \boldsymbol{K}, \Omega)
$$

is the noninteracting two-particle propagator which both in homogeneous matter and free space conserves the relative 
momentum as expressed by the $\delta$ function in Eq. (3). The presence of exchange terms in Eqs. (2) and (3) is hereby acknowledged but suppressed in the presentation. The second equality in Eq. (2) links the two-particle propagator with the vertex function or effective interaction $\Gamma$ which contains the summation of all ladder diagrams.

A partial-wave decomposition of the two-body propagator in Eq. (2) yields the corresponding integral equation and the relation between the propagator and the vertex function (assuming an appropriate angle-averaging procedure for nonzero total momentum)

$$
\begin{aligned}
g_{J S T}^{I I}\left(k l, k^{\prime} l^{\prime} ; K \Omega\right) & \\
= & \frac{\delta\left(k-k^{\prime}\right)}{k^{2}} \delta_{l, l^{\prime}} g_{f}^{I I}(k ; K, \Omega)+g_{f}^{I I}(k ; K, \Omega) \\
& \times \sum_{l^{\prime \prime}} \int d q q^{2}\left\langle k l\left|V^{J S T}\right| q l^{\prime \prime}\right\rangle g^{I I}\left(q l^{\prime \prime}, k^{\prime} l^{\prime} ; K, \Omega\right) \\
= & \frac{\delta\left(k-k^{\prime}\right)}{k^{2}} \delta_{l, l^{\prime}} g_{f}^{I I}(k ; K, \Omega)+g_{f}^{I I}(k ; K, \Omega) \\
& \times\left\langle k l\left|\Gamma^{J S T}(K, \Omega)\right| k^{\prime} l^{\prime}\right\rangle g_{f}^{I I}\left(k^{\prime} ; K, \Omega\right) .
\end{aligned}
$$

The appropriate notation for a partial wave basis has been introduced in Eq. (4) in terms of $l, S, J, T$ representing orbital, total spin, total angular momentum, and isospin, while $k$ and $k^{\prime}$ denote relative and $K$ total momentum quantum numbers. The energy $\Omega$ is conserved and must be viewed as a variable upon which the propagator depends (it also depends on the total momentum in the case of the medium). The noninteracting propagator is again denoted by $g_{f}^{I I}$ and may include the dressing of the individual particles when the scattering takes place in matter. The vertex function or effective interaction $\Gamma$ can be obtained from the numerical solution of the ladder equation in a partial-wave momentum representation

$$
\begin{aligned}
&\left\langle k l\left|\Gamma^{J S T}(K, \Omega)\right| k^{\prime} l^{\prime}\right\rangle \\
&=\left\langle k l\left|V^{J S T}\right| k^{\prime} l^{\prime}\right\rangle \\
&+\sum_{l^{\prime \prime}} \int_{0}^{\infty} d q q^{2}\left\langle k l\left|V^{J S T}\right| q l^{\prime \prime}\right\rangle g_{f}^{I I}(q ; K, \Omega) \\
& \times\left\langle q l^{\prime \prime}\left|\Gamma^{J S T}(K, \Omega)\right| k^{\prime} l^{\prime}\right\rangle .
\end{aligned}
$$

This equation has been solved for the first time using fully dressed sp propagators in the medium in Ref. [25]. Since this subsection deals primarily with general issues, the description of the calculation of the two-body interaction between dressed particles will be be deferred to Sec. II D. Suffice it to state here that it depends critically on an accurate evaluation of the dressed but noninteracting propagator $g_{f}^{I I}(q ; K, \Omega)$.

In order to extract the information relevant for the description of the scattering process from the effective twobody interaction or two-body propagator calculated in momentum space, it is necessary to consider both quantities in coordinate space. The coordinate space version of Eq. (4) is obtained by a double Fourier-Bessel transform

$$
\begin{aligned}
& g_{J S T}^{I I}\left(r l, r^{\prime} l^{\prime} ; K, \Omega\right) \\
&= \frac{2}{\pi} \int_{0}^{\infty} d k k^{2} \int_{0}^{\infty} d k^{\prime} k^{\prime 2} j_{l}(k r) j_{l^{\prime}}\left(k^{\prime} r^{\prime}\right) \\
& \times g_{J S T}^{I I}\left(k l, k^{\prime} l^{\prime} ; K, \Omega\right) .
\end{aligned}
$$

The corresponding result for the noninteracting part of the propagator, represented by the first term in Eq. (4), reduces to one integral on account of the delta function which conserves relative momentum:

$$
g_{f, l}^{I I}\left(r, r^{\prime} ; K, \Omega\right)=\frac{2}{\pi} \int_{0}^{\infty} d k k^{2} j_{l}(k r) j_{l}\left(k r^{\prime}\right) g_{f}^{I I}(k ; K, \Omega) .
$$

The Fourier-Bessel transform of Eq. (4) has the following form:

$$
\begin{aligned}
g_{J S T}^{I I}\left(r l, r^{\prime} l^{\prime} ; K, \Omega\right) & \\
= & \delta_{l, l^{\prime}} g_{f, l}^{I I}\left(r, r^{\prime} ; K, \Omega\right) \\
& +\sum_{l^{\prime \prime}} \int_{0}^{\infty} d r_{1} r_{1}^{2} \int_{0}^{\infty} d r_{2} r_{2}^{2} g_{f, l}^{I I}\left(r, r_{1} ; K, \Omega\right) \\
& \times\left\langle r_{1} l\left|V^{J S T}\right| r_{2} l^{\prime \prime}\right\rangle g_{J S T}^{I I}\left(r_{2} l^{\prime \prime}, r^{\prime} l^{\prime} ; K, \Omega\right) \\
= & \delta_{l, l^{\prime}} g_{f, l}^{I I}\left(r, r^{\prime} ; K, \Omega\right) \\
& +\int_{0}^{\infty} d r_{1} r_{1}^{2} \int_{0}^{\infty} d r_{2} r_{2}^{2} g_{f, l}^{I I}\left(r, r_{1} ; K, \Omega\right) \\
& \times\left\langle r_{1} l\left|\Gamma^{J S T}(K, \Omega)\right| r_{2} l^{\prime}\right\rangle g_{f, l^{\prime}}^{I I}\left(r_{2}, r^{\prime} ; K, \Omega\right) .
\end{aligned}
$$

When the bare two-body interaction $V$ is local in the relative coordinate, only one integral in the first equality remains. The second equality can be used to study the asymptotic behavior of the propagator outside the range of the interaction.

\section{B. Scattering of free particles}

The propagator description of the scattering of free particles was presented in Ref. [29] for the case of uncoupled channels. As a result of the importance of channel coupling related to the nuclear tensor force, it is important to present this case explicitly here. In the case of free particles the noninteracting propagator in momentum space is given by (assuming zero total momentum)

$$
g_{f}^{I I}(k ; \Omega)=\frac{1}{\Omega-\hbar^{2} k^{2} / m+i \eta} .
$$

Defining the on-shell momentum by

$$
\Omega=\frac{\hbar^{2} k_{0}^{2}}{m}
$$


one can perform the relevant Fourier-Bessel transform of the noninteracting propagator in Eq. (7) analytically (see, e.g., [32]), yielding

$$
g_{f, l}^{I I}\left(r, r^{\prime} ; k_{0}\right)=-i k_{0} \frac{m}{\hbar^{2}} j_{l}\left(k_{0} r_{<}\right) h_{l}\left(k_{0} r_{>}\right) .
$$

The coordinate argument in the spherical Hankel function must be the larger of $r$ and $r^{\prime}$ and is denoted by $r_{>}$, while the argument of the spherical Bessel function is the smaller and denoted by $r_{<}$. For the current analysis it will be assumed that the interaction has a finite range, $\left\langle r l\left|V^{J S T}\right| r^{\prime} l^{\prime}\right\rangle$ $=0$ for $r, r^{\prime}$ larger than some $r_{0}$. Substituting Eq. (11) in the second part of Eq. (8) in the case of coupled channels for $r^{\prime}>r$ and $r^{\prime}>r_{0}$ yields

$$
\begin{aligned}
g_{J S T}^{I I}( & \left.r l, r^{\prime} l^{\prime} ; k_{0}\right) \\
= & -\delta_{l, l^{\prime}} i k_{0} \frac{m}{\hbar^{2}} j_{l}\left(k_{0} r\right) h_{l^{\prime}}\left(k_{0} r^{\prime}\right) \\
& +\sum_{l^{\prime \prime}} \int_{0}^{\infty} d r_{1} r_{1}^{2} \int_{0}^{\infty} d r_{2} r_{2}^{2} g_{f, l}^{I I}\left(r, r_{1} ; k_{0}\right) \\
& \times\left\langle r_{1} l\left|T^{J S T}\left(k_{0}\right)\right| r_{2} l^{\prime \prime}\right\rangle\left(-i k_{0} \frac{m}{\hbar^{2}}\right) j_{l^{\prime \prime}}\left(k_{0} r_{2}\right) h_{l^{\prime}}\left(k_{0} r^{\prime}\right) \\
= & -i k_{0} \frac{m}{\hbar^{2}} \psi_{l^{\prime}}^{J S T}\left(r l ; k_{0}\right) h_{l^{\prime}}\left(k_{0} r^{\prime}\right),
\end{aligned}
$$

where

$$
\begin{aligned}
\psi_{l^{\prime}}^{J S T}\left(r l ; k_{0}\right)= & \delta_{l, l^{\prime}} j_{l}\left(k_{0} r\right)+\int_{0}^{\infty} d r_{1} r_{1}^{2} \int_{0}^{\infty} d r_{2} r_{2}^{2} g_{f, l}^{I I}\left(r, r_{1} ; k_{0}\right) \\
& \times\left\langle r_{1} l\left|T^{J S T}\left(k_{0}\right)\right| r_{2} l^{\prime}\right\rangle j_{l^{\prime}}\left(k_{0} r_{2}\right),
\end{aligned}
$$

and the conventional notation $T$ instead of $\Gamma$ has been introduced together with the replacement of $\Omega$ by $k_{0}$. This result can be substituted into the first part of Eq. (8) to obtain the relevant integral equation for the wave function $\psi$ (under the condition that $r^{\prime}>r_{0}$ ):

$$
\begin{aligned}
\psi_{l^{\prime}}^{J S T}\left(r l ; k_{0}\right)= & \delta_{l, l^{\prime}} j_{l}\left(k_{0} r\right) \\
& +\sum_{l^{\prime \prime}} \int_{0}^{\infty} d r_{1} r_{1}^{2} \int_{0}^{\infty} d r_{2} r_{2}^{2} g_{f, l}^{I I}\left(r, r_{1} ; k_{0}\right) \\
& \times\left\langle r_{1} l\left|V^{J S T}\right| r_{2} l^{\prime \prime}\right\rangle \psi_{l^{\prime}}^{J S T}\left(r_{2} l^{\prime \prime} ; k_{0}\right) .
\end{aligned}
$$

The asymptotic analysis of the wave function can be performed by using Eq. (11) in Eq. (13) assuming that $r$ is larger than $r_{0}$, the range of the interaction. Values of $r_{1}$ and $r_{2}$ in Eq. (8) larger than $r_{0}$ yield no contributions to the integral. As a result, the effective interaction $T$ has a range similar to the one of the bare interaction $V$. Using the relation between spherical Bessel and Hankel functions

$$
j_{l}(\rho)=\frac{1}{2}\left[h_{l}(\rho)+h_{l}^{*}(\rho)\right]
$$

and Eq. (11) in Eq. (13) one obtains the asymptotic behavior of the wave function for the general case of a coupled channel in the following form:

$$
\begin{aligned}
\psi_{l^{\prime}}^{J S T}\left(r l ; k_{0}\right) \rightarrow & \frac{1}{2}\left\{\delta_{l, l^{\prime}} h_{l}^{*}\left(k_{0} r\right)+h_{l}\left(k_{0} r\right)\right. \\
& \times\left[\delta_{l, l^{\prime}}-2 i \frac{m}{\hbar^{2}} k_{0} \int_{0}^{\infty} d r_{1} r_{1}^{2} \int_{0}^{\infty} d r_{2} r_{2}^{2}\right. \\
& \left.\left.\times\left\langle r_{1} l\left|T^{J S T}\left(k_{0}\right)\right| r_{2} l^{\prime}\right\rangle j_{l}\left(k_{0} r_{1}\right) j_{l^{\prime}}\left(k_{0} r_{2}\right)\right]\right\} \\
= & \frac{1}{2}\left\{\delta_{l, l^{\prime}} h_{l}^{*}\left(k_{0} r\right)+h_{l}\left(k_{0} r\right)\left[\delta_{l, l^{\prime}}-2 \pi i\left(\frac{m k_{0}}{2 \hbar^{2}}\right)\right.\right. \\
& \left.\left.\times\left\langle k_{0} l\left|T^{J S T}\left(k_{0}\right)\right| k_{0} l^{\prime}\right\rangle\right]\right\} .
\end{aligned}
$$

The asymptotic form of the propagator is obtained by inserting the result of Eq. (16) into Eq. (12). The term in square brackets corresponds to the possibly nondiagonal $S$-matrix element

$$
\begin{aligned}
\left\langle k_{0} l\left|S^{J S T}\left(k_{0}\right)\right| k_{0} l^{\prime}\right\rangle= & {\left[\delta_{l, l^{\prime}}-2 \pi i\left(\frac{m k_{0}}{2 \hbar^{2}}\right)\right.} \\
& \left.\times\left\langle k_{0} l\left|T^{J S T}\left(k_{0}\right)\right| k_{0} l^{\prime}\right\rangle\right] .
\end{aligned}
$$

In the case of an uncoupled channel one obtains the phase shift from

$$
e^{2 i \delta_{l}^{J S T}} \equiv\left\langle k_{0}\left|S_{l}^{J S T}\left(k_{0}\right)\right| k_{0}\right\rangle
$$

This result is equivalent to

$$
\tan \delta_{l}^{J S T}=\frac{\operatorname{Im}\left\langle k_{0}\left|T_{l}^{J S T}\left(k_{0}\right)\right| k_{0}\right\rangle}{\operatorname{Re}\left\langle k_{0}\left|T_{l}^{J S T}\left(k_{0}\right)\right| k_{0}\right\rangle},
$$

which explicitly shows that a nonzero imaginary part of the effective interaction is required to obtain a nonvanishing phase shift. In turn, this imaginary part of the interaction only appears for energies where the noninteracting propagator has a nonvanishing imaginary part. For the scattering of free particles this corresponds to all positive energies. In the case of coupled channels the unitarity of the $S$ matrix and the symmetry property of the $T$ matrix can be employed to infer that $S$ can be diagonalized by an orthogonal real matrix $A$ :

$$
\begin{aligned}
& \left\langle k_{0} l\left|S^{J S T}\left(k_{0}\right)\right| k_{0} l^{\prime}\right\rangle \\
& \quad=\sum_{\alpha=1,2}\left\langle l\left|A^{J}\left(k_{0}\right)\right| \alpha\right\rangle e^{2 i \delta_{\alpha}^{J S T}}\left\langle\alpha\left|A^{J}\left(k_{0}\right)\right| l^{\prime}\right\rangle,
\end{aligned}
$$


where $\delta_{\alpha}^{J S T}$ are called the (real) eigenphase shifts. One may choose [34]

$$
\left\langle l\left|A^{J}\left(k_{0}\right)\right| \alpha\right\rangle=\left(\begin{array}{rr}
\cos \epsilon^{J} & \sin \epsilon^{J} \\
-\sin \epsilon^{J} & \cos \epsilon^{J}
\end{array}\right),
$$

where $\epsilon^{J}$ is referred to as the mixing angle and the related mixing parameter is given by

$$
\rho^{J}=\sin 2 \epsilon^{J} .
$$

It should be noted that the three real parameters $\delta_{1}^{J S T}, \delta_{2}^{J S T}$, and $\epsilon^{J}$ can be used to represent the $S$ matrix. The relation of the eigenphase shifts and corresponding mixing parameter with the usual representation of the experimental results in terms of bar phase shifts can be found, e.g., in Ref. [35]. It is also possible to calculate the phase shifts and mixing parameter directly from the real $R$-matrix elements [36]. Since for the case of dressed nucleons the procedure corresponds to diagonalizing an $S$-matrix-like quantity, this method is highlighted here for the case of free particles.

\section{Scattering of mean-field particles in the medium}

To obtain the phase shifts for particles propagating in the medium with $\mathrm{mf} \mathrm{sp}$ energies one can proceed in a similar fashion. A useful reference is the work of Bishop et al. [31] where the introduction of the phase shift for the case of holehole propagation is discussed. The corresponding $\mathrm{mf}$ propagator in the medium, also known as the Galitski-Feynman propagator, is given by

$$
\begin{aligned}
g_{m f}^{I I}\left(k_{1}, k_{2} ; \Omega\right)= & \frac{\theta\left(k_{1}-k_{F}\right) \theta\left(k_{2}-k_{F}\right)}{\Omega-\epsilon\left(k_{1}\right)-\epsilon\left(k_{2}\right)+i \eta} \\
& -\frac{\theta\left(k_{F}-k_{1}\right) \theta\left(k_{F}-k_{2}\right)}{\Omega-\epsilon\left(k_{1}\right)-\epsilon\left(k_{2}\right)-i \eta},
\end{aligned}
$$

using the sp momenta $k_{1}$ and $k_{2}$. For the case of zero centerof-mass momentum which is the only one considered in Secs. III and IV, one obtains

$$
g_{m f}^{I I}(k ; \Omega)=\frac{\theta\left(k-k_{F}\right)}{\Omega-2 \epsilon(k)+i \eta}-\frac{\theta\left(k_{F}-k\right)}{\Omega-2 \epsilon(k)-i \eta} .
$$

The sp energy $\epsilon(k)$ can deviate from the simple kinetic energy spectrum and therefore yield a different relation between the energy $\Omega$ and the on-shell momentum $k_{0}$ :

$$
\Omega \equiv 2 \epsilon\left(k_{0}\right) .
$$

Nevertheless, the uniqueness of $k_{0}$ for a given energy is still preserved. Although one can no longer evaluate the noninteracting propagator in coordinate space completely analytically from Eq. (7), the separability of the propagator is maintained for the contribution of the pole term as in Eq. (11) (with a different constant prefactor), while the remaining term vanishes asymptotically for $r$ sufficiently different from $r^{\prime}$. A discussion of a similar result for the Fourier transform of the mf propagator given in Eq. (24) can be found in Ref. [37] for the Bethe-Goldstone propagator. As a result, one preserves the integral equation for the wave function in a partial wave basis as in Eq. (13) in the case of mf propagators. The only difference with the free scattering case involves the use of the $\mathrm{mf}$ equivalent of the noninteracting propagator in coordinate space in Eq. (14). This result is due to the uniqueness of the on-shell momentum at a given energy which guarantees that the noninteracting wave function is a plane-wave or spherical Bessel function (in a partialwave basis). One can therefore proceed with a similar asymptotic analysis as for free particles yielding a corresponding definition of the phase shifts as in Eq. (18) in terms of the on-shell scattering matrix. The result of Eq. (19) also remains valid in this case. For coupled channels Eq. (17) is still valid and eigenphase shifts are also obtained by diagonalizing the $S$ matrix. The presence of a nonvanishing phase shift continues to be linked to the nonvanishing of the imaginary part of the noninteracting propagator. In the case of $\mathrm{mf}$ scattering the corresponding energy domain resides above $2 \epsilon(k=0)$ which corresponds to the lowest energy of two occupied states.

As in the case of noninteracting particles, the presence of bound states has specific consequences for the behavior of the phase shift at the corresponding thresholds in the energy variable [31]. While in free space this threshold corresponds to zero energy and the presence of one bound state is reflected in the phase shift going to $\pi$ when the scattering energy goes to zero, the corresponding threshold in the medium is $2 \epsilon_{F}$. If the interaction is sufficiently attractive, the phase shift may approach $\pi$ on both sides of $2 \epsilon_{F}$. This feature is intimately related to the presence of a pairing instability or bound pair states in Fermi systems with attractive effective interactions at the Fermi surface. The phase shift can also approach $-\pi$ when a bound state below the holehole continuum [i.e., below $2 \epsilon(k=0)$ ] appears due to a repulsive interaction. This possibility is realized in liquid ${ }^{3} \mathrm{He}$ at sufficiently high density for mf particles [31,38]. Both cases (phase shifts going to $\pm \pi$ ) will be illustrated in Secs. III and IV by considering modifications of the ${ }^{1} S_{0}$ interaction of the Reid potential as well as the actual Reid ${ }^{1} S_{0}$ and ${ }^{3} S_{1}{ }^{3} D_{1}$ interactions. If the interaction is not sufficiently attractive to yield pairing, the phase shift will vanish at $2 \epsilon_{F}$.

The modesty of the modifications of the quantities that characterize the scattering process for mf particles as compared to the case of free-particle scattering, is related to the continued one-to-one relation of the energy with a unique relative momentum for which the noninteracting propagator has an imaginary part. This on-shell momentum emerges as the momentum that characterizes the spherical Bessel function describing the relative motion. The plane-wave character of the wave function allows for a conventional interpretation of the scattering process as in the case of free space.

\section{Scattering of dressed particles in the medium}

In order to discuss the modifications of the scattering process in the medium involving dressed nucleons it is useful to gather some general results involving the two-body propagator in the medium [Eq. (1)] and the two-body spectral density 
$S^{I I}\left(\boldsymbol{k}_{1} \boldsymbol{k}_{2} ; \boldsymbol{k}_{3} \boldsymbol{k}_{4} ; \Omega\right)$

$$
\begin{aligned}
= & -\frac{1}{\pi} \operatorname{Im} g^{I I}\left(\boldsymbol{k}_{1} \boldsymbol{k}_{2} ; \boldsymbol{k}_{3} \boldsymbol{k}_{4} ; \Omega\right) \\
= & -\frac{1}{\pi} \operatorname{Im} \int_{-\infty}^{\infty} d\left(t_{1}-t_{2}\right) e^{i \Omega\left(t_{1}-t_{2}\right)} \\
& \times\left\{-i\left\langle\Psi_{0}^{A}\left|T\left\{a_{\boldsymbol{k}_{2}}\left(t_{1}\right) a_{\boldsymbol{k}_{1}}\left(t_{1}\right) a_{\boldsymbol{k}_{3}}^{\dagger}\left(t_{2}\right) a_{\boldsymbol{k}_{4}}^{\dagger}\left(t_{2}\right)\right\}\right| \Psi_{0}^{A}\right\rangle\right\} .
\end{aligned}
$$

Since the total momentum of the added and removed pair must be the same on account of momentum conservation, it is more appropriate to consider the two-body spectral density and propagator without the corresponding $\delta$ function:

$$
S^{I I}\left(\boldsymbol{k}, \boldsymbol{k}^{\prime} ; \boldsymbol{K}, \Omega\right)=-\frac{1}{\pi} \operatorname{Im} g^{I I}\left(\boldsymbol{k}, \boldsymbol{k}^{\prime} ; \boldsymbol{K}, \Omega\right),
$$

where the relative momenta $\boldsymbol{k}, \boldsymbol{k}^{\prime}$ and the total momentum $\boldsymbol{K}$ are defined in a similar way as in the text below Eq. (1). $S^{I I}$ contains two contributions just as the two-body propagator in the second equality of Eq. (2):

$$
\begin{aligned}
S^{I I}\left(\boldsymbol{k}, \boldsymbol{k}^{\prime} ; \boldsymbol{K}, \Omega\right)= & -\frac{1}{\pi} \operatorname{Im}\left\{g_{f}^{I I}\left(\boldsymbol{k}, \boldsymbol{k}^{\prime} ; \boldsymbol{K}, \Omega\right)\right\} \\
& -\frac{1}{\pi} \operatorname{Im}\left\{g_{f}^{I I}(\boldsymbol{k} ; \boldsymbol{K}, \Omega)\left\langle\boldsymbol{k}|\Gamma(\boldsymbol{K}, \Omega)| \boldsymbol{k}^{\prime}\right\rangle\right. \\
& \left.\times g_{f}^{I I}\left(\boldsymbol{k}^{\prime} ; \boldsymbol{K}, \Omega\right)\right\} .
\end{aligned}
$$

The first part in Eq. (28) refers to the propagation of two dressed particles without their mutual interaction. This term contains a $\delta$ function as in Eq. (3) related to the conservation of the relative momentum since there is no mutual interaction. The spectral function corresponding to this noninteracting term is given by

$$
S_{f}^{I I}(\boldsymbol{k} ; \boldsymbol{K}, \Omega)=-\frac{1}{\pi} \operatorname{Im}\left\{g_{f}^{I I}(\boldsymbol{k} ; \boldsymbol{K}, \Omega)\right\}
$$

By returning to individual momenta for the removal or addition of individual particles one can write $g_{f}^{I I}(\boldsymbol{k} ; \boldsymbol{K}, \Omega)$ in terms of the sp spectral functions:

$$
\begin{aligned}
g_{f}^{I I}\left(k_{1}, k_{2} ; \Omega\right)= & \int_{\epsilon_{F}}^{\infty} d \omega \int_{\epsilon_{F}}^{\infty} d \omega^{\prime} \frac{S_{p}\left(k_{1}, \omega\right) S_{p}\left(k_{2}, \omega^{\prime}\right)}{\Omega-\omega-\omega^{\prime}+i \eta} \\
& -\int_{-\infty}^{\epsilon_{F}} d \omega \int_{-\infty}^{\epsilon_{F}} d \omega^{\prime} \frac{S_{h}\left(k_{1}, \omega\right) S_{h}\left(k_{2}, \omega^{\prime}\right)}{\Omega-\omega-\omega^{\prime}-i \eta} .
\end{aligned}
$$

Only the magnitude $k_{1}$ and $k_{2}$ are indicated here since there is no dependence of the sp spectral functions on the direction of the sp momentum. These particle and hole spectral functions $S_{p}$ and $S_{h}$, respectively, describe the distribution of the sp strength for a given momentum over the energy. They are continuous and have sizable peaks either above or below the Fermi energy, corresponding to a momentum state above or below $k_{F}$, at the so-called quasiparticle energy. For $k_{F}$ $=1.36 \mathrm{fm}^{-1}$, corresponding to normal density, the strength contained in the peak for momenta close to $k_{F}$ is typically only $70 \%[23,24,39]$. From the rest of the strength about $10 \%$ is found below the Fermi energy, another $10 \%$ in the first $100 \mathrm{MeV}$ above the Fermi energy, and the remaining $10 \%$ is spread thinly towards even higher energy as a result of the short-range and tensor correlations in the nuclear interaction [23]. First attempts to incorporate these features in the solution of the ladder equation have been explored in Refs. [25-28]. A critical ingredient in solving this problem is a careful evaluation of Eq. (30). First results of this evaluation can be found in Ref. [40]. The noninteracting twoparticle spectral function corresponding to Eq. (30) can be written as

$$
\begin{aligned}
& S_{f}^{I I}\left(k_{1}, k_{2} ; \Omega\right)=-\frac{1}{\pi} \operatorname{Im} g_{f}^{I I}\left(k_{1}, k_{2} ; \Omega\right) \\
& \quad= \begin{cases}\int_{\epsilon_{F}}^{\infty} d \omega S_{p}\left(k_{1}, \omega\right) S_{p}\left(k_{2}, \Omega-\omega\right), & \Omega>2 \epsilon_{F}, \\
\int_{-\infty}^{\epsilon_{F}} d \omega S_{h}\left(k_{1}, \omega\right) S_{h}\left(k_{2}, \Omega-\omega\right), & \Omega<2 \epsilon_{F} .\end{cases}
\end{aligned}
$$

It should be noted that for energies below $2 \epsilon_{F}$, Eq. (31) can also be written as

$$
\begin{aligned}
S_{f}^{I I}\left(k_{1}, k_{2} ; \Omega\right)= & \sum_{n}\left|\left\langle\Psi_{n}^{A-2}\left|a_{k_{1}} a_{k_{2}}\right| \Psi_{0}^{A}\right\rangle\right|^{2} \\
& \times \delta\left(\Omega-\left(E_{0}^{A}-E_{n}^{A-2}\right)\right) .
\end{aligned}
$$

Using the results of Eq. (31) one can obtain the following two sum rules:

$$
I_{>}^{I I}=\int_{2 \epsilon_{F}}^{\infty} d \Omega S_{f}^{I I}\left(k_{1}, k_{2} ; \Omega\right)=\left[1-n\left(k_{1}\right)\right]\left[1-n\left(k_{2}\right)\right]
$$

and

$$
I_{<}^{I I}=\int_{-\infty}^{2 \epsilon_{F}} d \Omega S_{f}^{I I}\left(k_{1}, k_{2} ; \Omega\right)=n\left(k_{1}\right) n\left(k_{2}\right),
$$

where $n(k)$ refers to the occupation of the sp momentum $k$ which is related to the integral over energy of the hole spectral function

$$
n(k)=\int_{-\infty}^{\epsilon_{F}} d \omega S_{h}(k, \omega) .
$$

The sum rule [Eq. (34)] may yield large deviations from the free-Fermi-gas result. Using the numbers of Ref. [39] for $k_{F}=1.36 \mathrm{fm}^{-1}$ yields about $(0.8)^{2}$ for Eq. (34) when both momenta are below $k_{F}$ as compared to unity in the case of 


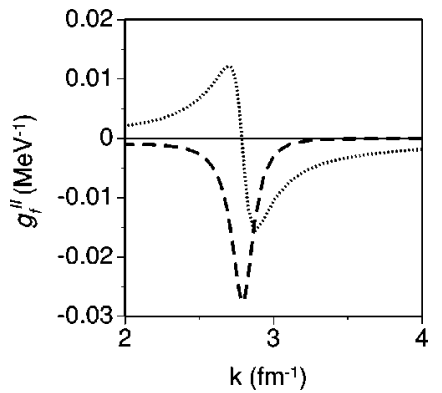

FIG. 1. Real (dotted line) and imaginary (dashed line) parts of the dressed noninteracting propagator for an "on-shell" momentum of $2.8 \mathrm{fm}^{-1}$ corresponding to an energy of $281 \mathrm{MeV}$, as a function of momentum. The propagator is considered at zero total momentum.

the free Fermi gas. Another important quantity related to the noninteracting two-body spectral function is the density of states which is given by

$$
N^{(2)}(K, \Omega)=\int_{0}^{\infty} d k k^{2} S_{f}^{I I}(k ; K, \Omega),
$$

where the magnitudes of the relative and total momentum are used assuming an appropriate angle-averaging procedure. This result may be compared to the result for a free Fermi gas for zero total momentum:

$$
N_{F}^{(2)}(\Omega)=\frac{m^{3 / 2} \Omega^{1 / 2}}{2 \hbar^{3}}
$$

Using the unique relation between the energy and the onshell momentum $k_{0}$ [see Eq. (10)] this result can be written as

$$
N_{F}^{(2)}\left(k_{0}\right)=\frac{m k_{0}}{2 \hbar^{2}}
$$

In the case of mf particles in the medium one has to include the effect of the sp potential energy $U$ which yields the following density of states (again for zero total momentum):

$$
N_{m f}^{(2)}\left(k_{0}\right)=\frac{m k_{0}^{2}}{2 \hbar^{2}} \frac{1}{k_{0}+\left(m / \hbar^{2}\right) \partial U /\left.\partial k\right|_{k_{0}}} .
$$

For purposes of comparison it is convenient to consider the density of states for dressed particles also as a function of momentum. This is achieved for zero total momentum by determining an "on-shell" momentum by

$$
\Omega=2 \epsilon\left(k_{0}\right)=2\left\{\frac{\hbar^{2} k_{0}^{2}}{2 m}+\operatorname{Re} \Sigma\left(k_{0}, \epsilon\left(k_{0}\right)\right)\right\},
$$

where $\Sigma$ corresponds to the sp self-energy. Except for energies deep in the Fermi sea [29] this on-shell momentum $k_{0}$ coincides with the location of the peak in the imaginary part of $g_{f}^{I I}$ as a function of momentum. This is shown in Fig. 1 for $k_{0}=2.8 \mathrm{fm}^{-1}$ corresponding to an energy of $281 \mathrm{MeV}$. For

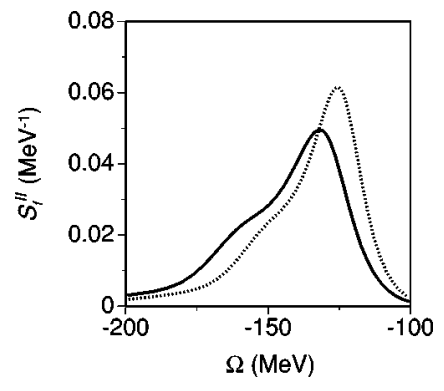

FIG. 2. Two-nucleon spectral function for two different momenta corresponding to $0.5 \mathrm{fm}^{-1}$ (solid line) and $0.6 \mathrm{fm}^{-1}$, respectively, as a function of energy. In both cases the total momentum is zero. The actual peak for both curves lies a few $\mathrm{MeV}$ below the corresponding energies as determined by Eq. (40).

the case of zero total momentum [implying that the magnitude of the sp momenta in Eq. (30) is the same] both the real (dotted line) and imaginary part (dashed line) parts of the dressed propagator are plotted for this fixed energy (and "on-shell" momentum) as a function of momentum. Note that for zero total momentum the relative momentum and $\mathrm{sp}$ momentum appearing in Eq. (30) are identical. The position of the peak in the imaginary part clearly corresponds to the on-shell momentum for this energy. In contrast to a mf propagator which has a $\delta$-function imaginary part at 2.8 $\mathrm{fm}^{-1}$, the dressed propagator displays a distribution over momentum at a given energy. Also for the real part of the propagator there is a distinct difference between the $\mathrm{mf}$ and dressed propagators. While the mf propagator jumps from $+\infty$ to $-\infty$ at $2.8 \mathrm{fm}^{-1}$, the dressed propagator exhibits a characteristic wiggle around this energy going now through zero at the "on-shell" momentum. For other momenta in Fig. 1 one obtains a reduction factor of about 0.5 with respect to the mf result, while only for large values of $k$ do the real parts approach each other [29]. Figure 1 also suggests that the numerical solution to Eq. (5) requires a somewhat different strategy than in the $\mathrm{mf}$ case. For $\mathrm{mf}$ propagators one usually solves Eq. (5) by discretizing the integral equation, taking only the real part of the propagator into account. The solution to this integral equation then yields the $R$ matrix by a real matrix inversion [36]. The contribution of the imaginary part of the propagator can then be obtained algebraically using the $R$-matrix elements [41]. Using dressed propagators it is more convenient to discretize the integral equation in such a way that the relevant sampling of both the real and imaginary parts of the propagator occurs simultaneously, leading to a complex matrix inversion to obtain $\Gamma$ directly.

For small on-shell momenta it is possible that the peak in the imaginary part of the propagator (or equivalently in $S_{f}^{I I}$ ) does not coincide with the "on-shell" momentum. This result is indicated in Fig. 2 where $S_{f}^{I I}$ is plotted as a function of energy for two different (relative) momenta (still for zero total momentum). In both cases the actual peak occurs a few $\mathrm{MeV}$ below the energy obtained from Eq. (40). The results in Fig. 2 also imply that for fixed energy, but now as a function of momentum, the peak in the spectral function does not always occur at the on-shell momentum but typically at a 


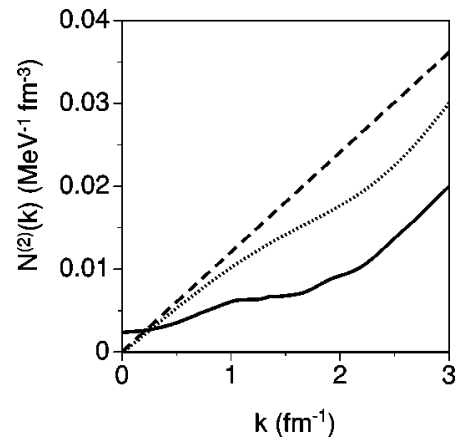

FIG. 3. Density of two-particle states as a function of the onshell momentum for free particles (dashed line), for mf particles at $k_{F}=1.36 \mathrm{fm}^{-1}$, including a sp spectrum $U$ (dotted line), and for dressed particles (solid line). All three lines correspond to zero total momentum. The dashed line also represents the density of states for $\mathrm{mf}$ particles in the medium when only kinetic energies are considered.

somewhat higher value. This is true in particular for energies deep in the Fermi sea. This observation points to the general feature that there is no unique on-shell momentum when one deals with dressed particles. As a result it is not obvious that one can use expressions like Eq. (19) to determine the phase shifts.

Before discussing the determination of the phase shifts for dressed particles it is useful to further characterize the difference between the noninteracting $\mathrm{mf}$ and dressed propagator. In Fig. 3 the density of states for these different cases is considered. The dashed line represents the result for free particles (or mf particles with only kinetic energy) according to Eq. (38). The dotted line shows the effect of a realistic sp spectrum [39] for mf particles at $k_{F}=1.36 \mathrm{fm}^{-1}$ and uses Eq. (39). The inclusion of the complete dressing leads to the solid line in Fig. 3 based on the evaluation of Eq. (36). When the on-shell momentum approaches $k_{F}$ the reduction of the density of states compared to the dotted line corresponds exactly to a factor $z_{k_{F}}^{2}$ (about 0.5 ), corresponding to a reduction of the strength of the quasiparticle pole at $k_{F}$ for each of the particles. Figure 3 shows that this reduction is substantial in a large domain of momenta (or equivalently in a large domain of energies) corresponding to the redistribution of the sp strength over a large energy domain [23]. For small momenta the dressed density of states does not go to zero

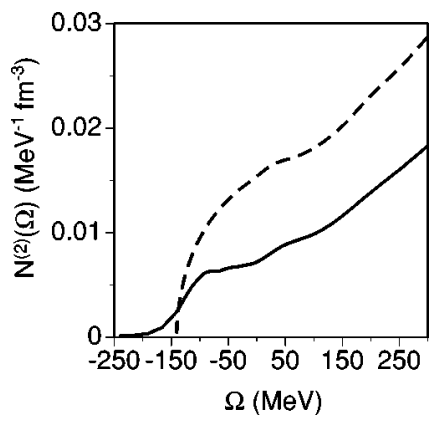

FIG. 4. Density of states as a function of energy for mf particles (dashed line) and dressed particles (solid line). since the on-shell relation (40) is no longer applicable for energies below $2 U(k=0)$ while the density of states does not vanish at those energies. This feature is demonstrated more clearly in Fig. 4 where the dressed density of states (solid line) is plotted as a function of energy and compared with the result for $\mathrm{mf}$ particles including the sp spectrum (dashed line) which starts at $2 U(0)$ corresponding to about $-140 \mathrm{MeV}$ at this density.

It should be noted that the noninteracting propagator in Eq. (30) becomes the familiar mf Galitski-Feynman propagator [see Eq. (24)] when $\mathrm{mf}$ spectral functions are inserted which are characterized by a $\delta$-function peak of strength 1 at a sp energy either above the Fermi energy $\left(k>k_{F}\right)$ or below $\left(k<k_{F}\right)$. The difference between the Galitski-Feynman propagator and the dressed propagator is qualitatively different for the imaginary part and quantitatively for the real part as discussed in Ref. [29] and above with regard to Fig. 1. It was shown in Ref. [29] that the spreading over a wide range of momenta of the imaginary part of the propagator at a given energy is responsible for the localization of the scattering process. This feature was demonstrated analytically by approximating the noninteracting dressed propagator in terms of a propagator which has a simple pole in the complex momentum plane. Using this complex pole approximation (CPA) an asymptotic analysis of the scattering process was made in Ref. [29]. The critical ingredient in this analysis is the new form of the dressed propagator in coordinate space in this CPA. It is obtained from Eq. (11) by replacing $k_{0}$ by the complex momentum corresponding to the pole of the CPA and inserting an overall multiplication factor $c$ [29]. The imaginary part of this pole momentum characterizes the width of the imaginary part of the propagator on the real momentum axis as in Fig. 1. Its presence is responsible for the finite range of the propagator in coordinate space since the probability amplitude for removing a pair with relative distance $r$ while adding it after propagation at $r^{\prime}$ is exponentially damped, its decay governed by this imaginary part of the momentum.

One consequence of this damping is the absence of a formal definition of the cross section, since this requires asymptotically large distances for flux to arrive at a detector. The dressing of particles in the medium simply implies that after scattering the particles do not retain information about this scattering event over asymptotically large distances since they will interact with other particles while propagating in the medium. The above discussion does not imply that the local interaction between dressed particles is small. It does mean that one has to be cautious with the notion of a cross section of dressed particles in the medium.

Another consequence of this damping feature is the appearance of complex phase shifts as illustrated in Ref. [29] for the case of a hard-core potential. Fortunately this imaginary part of the phase shift is in practice substantially smaller than the real part since their relative size is related to the relative size of the real and imaginary parts of the complex momentum that characterizes the spread in momentum of the propagator. For most energies the imaginary part of this momentum is substantially smaller than the real part. An example of this feature is provided in Fig. 1 where the real part 
of the pole momentum is close to $2.8 \mathrm{fm}^{-1}$ (the "on-shell", value) while the imaginary part is approximately $0.2 \mathrm{fm}^{-1}$. In order to facilitate the comparison with mf calculations of phase shifts and cross sections, it is therefore useful to find approximate expressions for real phase shifts and corresponding cross sections that still reflect the spread in momentum of the dressed propagator at a given energy. Such expressions have been proposed in Ref. [29]. They are based on the smallness of the imaginary part of the complex momentum in the CPA which suggests that it makes sense to make use of the formal identity for the free propagator (for $r<r^{\prime}$ ) that is part of Eq. (16):

$$
\begin{aligned}
& -i k_{0} j_{l}\left(k_{0} r\right) j_{l}\left(k_{0} r^{\prime}\right) \\
& =\frac{-i k_{0}}{2}\left\{j_{l}\left(k_{0} r\right) h_{l}\left(k_{0} r^{\prime}\right)+j_{l}\left(k_{0} r\right) h_{l}^{*}\left(k_{0} r^{\prime}\right)\right\} \\
& =\frac{1}{\pi} \int_{0}^{\infty} d k k^{2} \frac{j_{l}(k r) j_{l}\left(k r^{\prime}\right)}{k_{0}^{2}-k^{2}+i \eta}-\frac{1}{\pi} \int_{0}^{\infty} d k k^{2} \frac{j_{l}(k r) j_{l}\left(k r^{\prime}\right)}{k_{0}^{2}-k^{2}-i \eta} \\
& =i \frac{2}{\pi} \int_{0}^{\infty} d k k^{2} j_{l}(k r) j_{l}\left(k r^{\prime}\right) \operatorname{Im}\left\{\frac{1}{k_{0}^{2}-k^{2}+i \eta}\right\},
\end{aligned}
$$

which is valid for vanishing $\eta$, in the case of the dressed propagator. By identifying in the last equality of Eq. (41) the appearance of the imaginary part of the propagator (for the free case), one may extend this result to the case of the CPA propagator or the complete dressed propagator. This approximation is appropriate for a pole in the complex momentum plane not too far from the real axis for the CPA result but also makes sense for $r$ no too different from $r^{\prime}$ in general. Using this extension of the last equality in Eq. (41) to the dressed propagator, the $S$-matrix element can be written [see Eqs. (16) and (17)] for the case of zero total momentum in the following way:

$$
S_{l, l^{\prime}}^{J S T}(\Omega)=1+2 i \int_{0}^{\infty} d k k^{2} \operatorname{Im}\left\{g_{f}^{I I}(k ; \Omega)\right\}\left\langle k l\left|\Gamma^{J S T}(\Omega)\right| k l^{\prime}\right\rangle .
$$

This result reduces to the conventional results [see, e.g., Eq. (17)] for free or mf particles. In the case of coupled channels Eq. (42) can be used to follow the procedure to obtain phase shifts by diagonalization as discussed in Sec. II B. In the case of an uncoupled channel one can define the dressed phase shift according to

$$
\begin{aligned}
S_{l, l}^{J S T}(\Omega) & =1+2 i \int_{0}^{\infty} d k k^{2} \operatorname{Im}\left\{g_{f}^{I I}(k ; \Omega)\right\}\left\langle k l\left|\Gamma^{J S T}\right| k l\right\rangle \\
& \equiv e^{2 i \delta_{l}^{J S T}} .
\end{aligned}
$$

A consequence of the present approximation is that the phase shifts $\delta_{l}^{J S T}$ remain real [29]. As a result, the phase shifts can be fruitfully compared with results for mf or free particles. Detailed results for a realistic interaction will be presented in Sec. IV. Equation (42) is exact for noninteracting or mf particles and for dressed particles includes the physically rea- sonable expectation that the distribution over the momenta as contained in the imaginary part of the propagator will feature in determining the scattering process. While this approximation does not make sense at large distance scales, it provides, locally, a very reasonable generalization of the phase shift. The corresponding "short-distance" approximation to the scattering amplitude yields the following result [29]:

$$
\begin{aligned}
f_{m_{s}^{\prime} m_{s}}^{S}(\theta, \phi)= & 4 \pi \sum_{l l^{\prime} J} \sum_{m m^{\prime} M} i^{l^{\prime}}(-i)^{l} Y_{l m_{l}}(\hat{\mathbf{r}}) Y_{l^{\prime} m_{l}^{\prime}}^{*}(\hat{\mathbf{z}}) \\
& \times\left(l m_{l} S m_{s} \mid J M\right)\left(l^{\prime} m_{l}^{\prime} S m_{s}^{\prime} \mid J M\right) \\
& \times \int_{0}^{\infty} d k k \operatorname{Im}\left\{g_{f}^{I I}(k ; \Omega)\right\} \\
& \times\left\langle k(l S) J|\Gamma(\Omega)| k\left(l^{\prime} S\right) J\right\rangle
\end{aligned}
$$

where a coupling to total spin $S$ and projections $m_{s}, m_{s}^{\prime}$ for initial and final spin states has been included together with the usual decomposition in partial waves. In the case of free or mf particle scattering the $\delta$ function of the imaginary part of $g_{f}^{I I}$ yields the conventional result. For the case of a central interaction and free particles Eq. (44) reduces to (suppressing spin indices)

$$
\begin{aligned}
f(\theta, \phi) & =\sum_{l} \frac{2 l+1}{k_{0}}\left\{\frac{-m k_{0} \pi}{2 \hbar^{2}}\right\}\left\langle k_{0}\left|T_{l}\left(k_{0}\right)\right| k_{0}\right\rangle P_{l}(\cos \theta) \\
& =\sum_{l} \frac{2 l+1}{k_{0}} e^{i \delta_{l}} \sin \delta_{l} P_{l}(\cos \theta),
\end{aligned}
$$

where the addition theorem for spherical harmonics and the $\delta$ function of the imaginary part of the propagator have been used to obtain the first equality and Eqs. (17) and (18) to obtain the second equality of this result. For the total cross section (in the neutron-proton case) one obtains

$$
\begin{aligned}
\sigma_{\text {tot }}= & \pi \sum_{S l l^{\prime} J}(2 J+1) \mid \int_{0}^{\infty} d k k \operatorname{Im}\left\{g_{f}^{I I}(k ; \Omega)\right\} \\
& \times\left.\left\langle k(l S) J|\Gamma(\Omega)| k\left(l^{\prime} S\right) J\right\rangle\right|^{2},
\end{aligned}
$$

which for a central interaction and free particles reduces to the standard result

$$
\sigma_{\mathrm{tot}}=\frac{4 \pi}{k_{0}^{2}} \sum_{l}(2 l+1) \sin ^{2} \delta_{l}
$$

Equation (46) demonstrates that a sensible cross section will be obtained in the case of dressed particles at all energies for which a nonvanishing imaginary part of the propagator exists. For two particles deep in the Fermi sea, for example, Eq. (46) avoids the divergence associated with the $k_{0}^{-2}$ term in Eq. (47). The formulation of the cross section in terms of Eq. (46) provides a reasonable way to assess the strength of the interaction between dressed particles in the medium in terms of the square of the relevant transition matrix element $(\Gamma)$ 


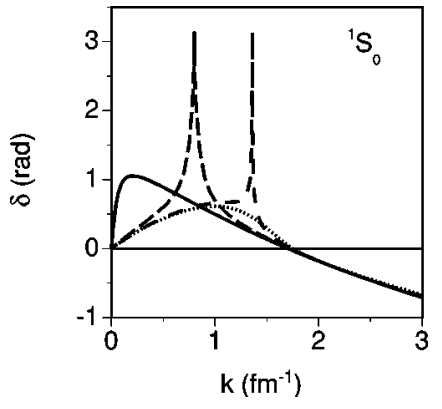

FIG. 5. Phase shift for the ${ }^{1} S_{0}$ channel of the Reid potential at various densities as a function of the on-shell momentum. Both for free particles (solid line) and mf particles in the medium corresponding to $k_{F}=0.8$ (long-dashed line), 1.36 (dashed line), and 1.8 $\mathrm{fm}^{-1}$ (dotted line) a kinetic energy spectrum was used.

multiplied by an appropriate measure of the density of states represented by the imaginary part of the noninteracting propagator [see also Eq. (36)].

\section{RESULTS FOR MEAN-FIELD PARTICLES}

The results discussed in this section involve the propagation of $\mathrm{mf}$ particles in nuclear matter at zero temperature. The aim is to exhibit some characteristic changes that occur in the medium for the phase shifts of the most important channels in the $N N$ interaction with respect to their behavior in free space. The interaction used is the Reid soft-core interaction [30]. An additional goal is to make contact with the discussion of Ref. [31] where the determination of phase shifts for the Galitski-Feynman propagator was introduced for a central interaction appropriate for ${ }^{3} \mathrm{He}$ atoms. This goal will be achieved by suitably modifying the ${ }^{1} S_{0}$ channel of the Reid interaction and studying the density dependence of the resulting phase shifts. The discussion in this section also prepares for the comparison with the results obtained for propagating dressed particles.

In Fig. 5 the phase shift for the ${ }^{1} S_{0}$ channel is shown as a function of the on-shell momentum for various densities and compared with the result in free space (solid line). The longdashed, dashed, and dotted lines correspond to Fermi momenta of $0.8,1.36$, and $1.8 \mathrm{fm}^{-1}$, respectively. For simplicity and ease of comparison a sp spectrum of kinetic energy was assumed in obtaining these results. The on-shell momentum was used as the plotting variable in Fig. 5 instead of the energy since it allows a direct comparison between results for free and $\mathrm{mf}$ particles at different densities. While the nuclear interaction in the ${ }^{1} S_{0}$ channel is not sufficiently attractive to generate a bound state in free space, it is sufficiently attractive in the medium to yield a pairing solution in a wide range of densities (see, e.g., Ref. [42]). The presence of a pairing solution can be inferred from the behavior of the phase shifts. When bound pair states in the effective interaction occur, it implies that the phase shift at the corresponding energy threshold (in this case $2 \epsilon_{F}$ ) will tend to $\pi$ [31]. This result is indeed exhibited for the phase shifts corresponding to $k_{F}=0.8$ and $1.36 \mathrm{fm}^{-1}$. In both cases the phase shift on either side of $2 \epsilon_{F}$ (or, as in Fig. 5, on either side of $k_{F}$ )

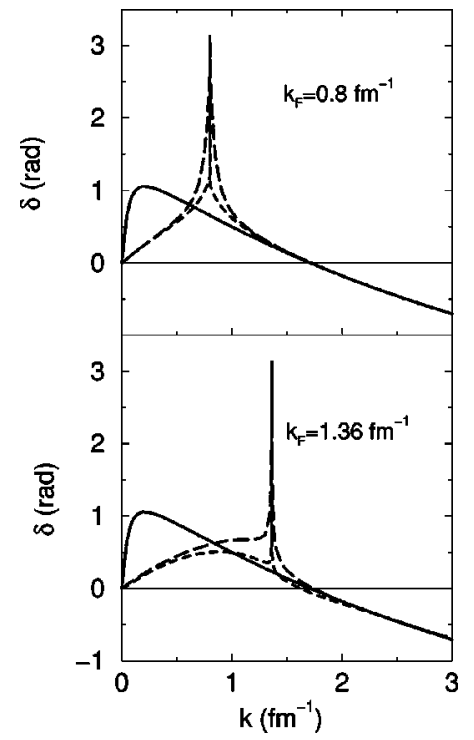

FIG. 6. Sensitivity of the ${ }^{1} S_{0}$ phase shift in the medium to a gap in the sp spectrum at the Fermi momentum for $k_{F}=0.8 \mathrm{fm}^{-1}$ (top panel) and the inclusion of a realistic sp spectrum for $k_{F}$ $=1.36 \mathrm{fm}^{-1}$ (bottom panel).

approaches $\pi$. It is also clear from Fig. 5 that the phase shift tends to $\pi$ more abruptly for $k_{F}=1.36 \mathrm{fm}^{-1}$ than for 0.8 $\mathrm{fm}^{-1}$, while it no longer does so for $k_{F}=1.8 \mathrm{fm}^{-1}$. These results correspond closely to the appearance and strength of bound pair states as obtained in Ref. [43] where they acquire the largest binding at $k_{F}=0.8 \mathrm{fm}^{-1}$, almost no binding at $1.36 \mathrm{fm}^{-1}$, and no bound states exist at $1.8 \mathrm{fm}^{-1}$. Indeed, the density range corresponding to the appearance of bound pair states exactly corresponds to the appearance of a phase shift of $\pi$ at $k_{F}$. This observation is commensurate with the suggestion that positive phase shifts in the medium near $k_{F}$ $\left(2 \epsilon_{F}\right)$, and therefore an attractive effective interaction, indicate the presence of bound pair states. The general behavior around $k_{F}$ is then an indication of the amount of correlation, strong pairing indicated by a phase shift that is already large and positive quite far away from $k_{F}$.

The sensitivity of the ${ }^{1} S_{0}$ phase shift to the sp spectrum or a gap in the sp spectrum at $k_{F}$ is explored in Fig. 6. In the top panel the kinetic energy spectrum at $k_{F}=0.8 \mathrm{fm}^{-1}$ was modified by including a $7 \mathrm{MeV}$ gap between sp states above and below the Fermi momentum. This gap ensures that the eigenvalues of the bound pair states fall inside the corresponding $14 \mathrm{MeV}$ gap in the two-particle spectrum [43] and are therefore real. When a pure kinetic energy spectrum is used, these eigenvalues acquire complex values, indicating the pairing instability. The dashed line in the top panel refers to the kinetic energy spectrum and the short-dashed line includes the gap in the spectrum. In order to understand these results it is useful to remember that the ladder equation including both particle-particle and hole-hole propagation is equivalent to a random phase approximation (RPA) summation. While the phase shift in the top panel of Fig. 6 still tends to $\pi$ at $k_{F}$ when the gap in the sp spectrum is introduced, it is also clear that the reduction of the RPA collectivity by the introduction of this gap between particle and 


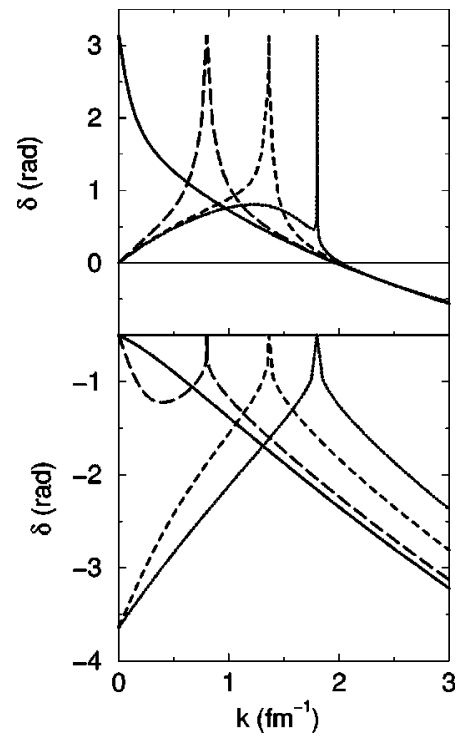

FIG. 7. Phase shifts obtained for modified versions of the Reid ${ }^{1} S_{0}$ interaction. In the top panel the intermediate range attraction is increased by $10 \%$. Phase shifts for the same densities as in Fig. 5 illustrate that this modified interaction yields a bound state for free particles which is illustrated by the corresponding phase shift going to $\pi$ at zero momentum (solid line). For the highest density $\left(k_{F}\right.$ $=1.8 \mathrm{fm}^{-1}$ ) the phase shift at $k_{F}$ tends to $\pi$ now in contrast to the result shown in Fig. 5. In the bottom panel the shortest-range Yukawa of the Reid ${ }^{1} S_{0}$ interaction is multiplied by a factor of 10 to simulate an atom-atom-like interaction. Results are shown for the same set of densities as in the top panel.

hole states leads to a less attractive phase shift around $k_{F}$. A similar reduction of the attraction exhibited by the phase shift is observed in the lower panel of Fig. 6 corresponding to $k_{F}=1.36 \mathrm{fm}^{-1}$ when a realistic sp potential energy $[39,40]$ is added. The dashed line in the bottom panel of Fig. 6 corresponds to the kinetic energy spectrum while the shortdashed line includes the sp potential energy. Also in this case the average distance between particle and hole energies is enlarged by the sp potential energy which reduces the collectivity of the RPA summation in the ladder equation.

Another illustration of the connection between the behavior of the phase shift near $k_{F}$ and bound pair states is provided in the top panel of Fig. 7. This panel demonstrates the behavior of the phase shifts for the same set of densities as in Fig. 5 but for a modified version of the Reid ${ }^{1} S_{0}$ interaction. By multiplying the intermediate-range attraction of this three-Yukawa interaction by a factor of 1.1, one already generates a bound state in free space reflected by the corresponding phase shift going to $\pi$ at zero momentum in the top panel of Fig. 7 (solid line). The other three curves correspond to the same set of Fermi momenta as in Fig. 5 (again using a kinetic energy sp spectrum in the medium). Comparing the phase shifts for these two interactions at the same density one observes, as expected, a substantially more positive phase shift for the more attractive interaction. For the highest Fermi momentum $\left(k_{F}=1.8 \mathrm{fm}^{-1}\right)$ the more attractive interaction now has a phase shift of $\pi$ at $k_{F}$ unlike the actual Reid ${ }^{1} S_{0}$ interaction, demonstrating that the range of densities where pairing occurs is enlarged.
The effective ${ }^{1} S_{0}$ interaction in the nuclear medium is sufficiently attractive to yield (at least when propagating $\mathrm{mf}$ particles) a paired ground state in a wide range of densities. As discussed above one may reach this conclusion also by inspecting whether the phase shift at $k_{F}$ (or equivalently 2 $\epsilon_{F}$ ) tends to $+\pi$. It is also instructive to illustrate the condition under which the phase shift from the GalitskiFeynman integral equation tends to $-\pi$. In earlier papers $[31,38]$ it was shown that this case occurs when a hole-hole bound state exists below the hole-hole continuum, which corresponds to higher excitation energies than can be obtained by removing two mf particles. Such a spectrum is generated by propagating $\mathrm{mf}{ }^{3} \mathrm{He}$ atoms in the medium interacting by means of a realistic atom-atom interaction. In order to simulate this type of interaction, one may increase the strength of the short-range repulsion of the Reid ${ }^{1} S_{0}$ channel by a factor of 10 . The corresponding phase shift results for the same set of densities are shown in the bottom panel of Fig. 7. The phase shift for free particles now always indicates a repulsive effective interaction (negative phase shift). For $k_{F}=0.8 \mathrm{fm}^{-1}$ the phase space of the hole-hole continuum is not yet large enough to yield a bound state, whereas for $k_{F}=1.36$ and $1.8 \mathrm{fm}^{-1}$ this is the case, yielding a phase shift of $-\pi$ at the corresponding energy threshold at zero momentum which is associated with the highest twohole excitation energy that the mf picture allows.

Results for other channels will be mentioned while comparing with the results for the propagation of dressed particles. It is appropriate to mention, however, that the present results are consistent with the results obtained in Ref. [13] to finite temperature. One difference that should be noted corresponds to the different boundary condition that is applied in Ref. [13] for the propagation of hole-hole states. As a result, the corresponding phase shifts differ from the ones obtained here and in Refs. [31] and [38] by a sign. In addition, the calculations in Ref. [13] have been performed above the critical temperature for pairing. This choice implies that the effective interaction at twice the chemical potential (the generalization of $2 \epsilon_{F}$ at finite temperature) is repulsive yielding a phase shift of zero at this energy (instead of $\pi$ in most cases considered here for the ${ }^{1} S_{0}$ and ${ }^{3} S_{1}$ channels).

\section{RESULTS FOR DRESSED NUCLEONS}

The proposed expressions for phase shifts and cross sections for dressed particles discussed in Sec. II D and originally proposed in Ref. [29] involve the folding of the imaginary part of the dressed but noninteracting propagator with the effective interaction. While this result yields the proper limit for $\mathrm{mf}$ or free particles, it is important to compare the result obtained with Eq. (43) with results obtained by attempting to define an "on-shell" momentum for a given energy as in Eq. (40) and then using Eq. (19). Such a comparison is made in Fig. 8 for the ${ }^{1} S_{0}$ phase shift. The solid line corresponds to Eq. (43) while the dotted line corresponds to the on-shell momentum definition. Comparison of both definitions for the dressed phase shift indicates that no serious difference exists in a wide energy domain. A more critical difference is encountered when cross sections are 


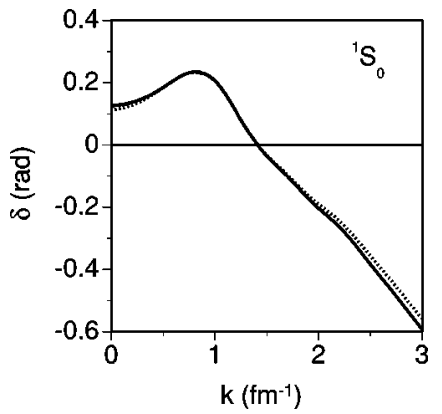

FIG. 8. Comparison of the ${ }^{1} S_{0}$ phase shift for dressed particles obtained by folding the effective interaction over the imaginary part of the dressed propagator using Eq. (43) (solid line) and the phase shift obtained by using Eq. (19) for the "on-shell" momentum given by Eq. (40) (dotted line).

considered (see below). It should be noted that particularly around $k_{F}$, or equivalently $2 \epsilon_{F}$, there cannot be any difference between both definitions since in this limit the imaginary part of the dressed propagator tends to a $\delta$ function located at $2 \epsilon_{F}$ and therefore yields an identical result to the "on-shell" definition. While both definitions of the phase shift do not differ very dramatically for those values of the energy for which Eq. (40) yields an actual solution for the momentum, it should be kept in mind that no such solution exists for energies below twice the lowest quasiparticle energy. Since the strength distributions of the spectral functions do not vanish abruptly at this energy, it is evident that below this energy only Eq. (43) makes sense. The corresponding result for the phase shift as a function of energy is displayed in Fig. 9. Below the on-shell threshold energy corresponding to $-140 \mathrm{MeV}$ the phase shift further decreases smoothly to zero, reflecting the decrease of the density of states shown in Fig. 4.

The main results for the phase shifts for some of the more important partial wave channels are summarized in Fig. 10. A comparison is made between phase shifts for free particles (solid line), mf particles at $k_{F}=1.36 \mathrm{fm}^{-1}$ (dashed line), and dressed particles (short-dashed line) at the same density for the ${ }^{1} S_{0},{ }^{3} S_{1},{ }^{3} P_{1}$, and ${ }^{3} D_{1}$ channels (corresponding to

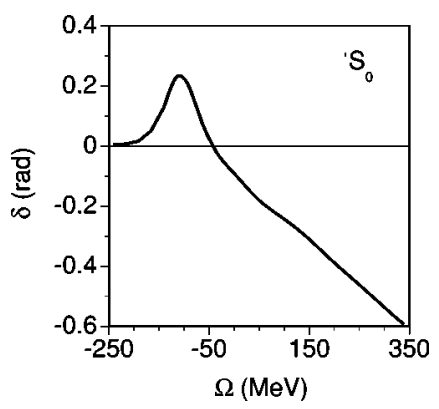

FIG. 9. Result for the dressed ${ }^{1} S_{0}$ phase shift as a function of energy. Below about $-140 \mathrm{MeV}$ there is no solution for the onshell momentum defined by Eq. (40). The result obtained by folding the effective interaction with imaginary part of the propagator (spectral function) yields a smooth decline to zero when the energy decreases below this threshold reflecting the reduction in the density of states shown in Fig. 4.

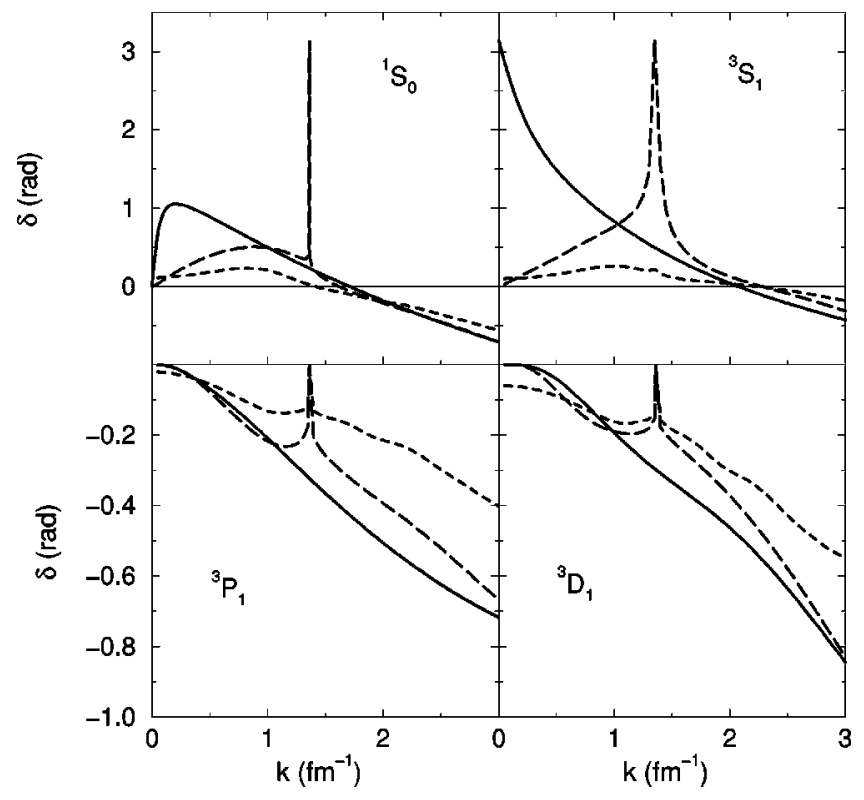

FIG. 10. Comparison of phase shifts for free particles (solid line), mf particles (dashed line), and dressed particles (short-dashed line) for different partial waves. The density of the medium corresponds to $k_{F}=1.36 \mathrm{fm}^{-1}$.

the different panels in Fig. 10) as a function of the on-shell momentum. In general one finds that the dressed phase shifts suggest weaker interactions since in essentially all cases they are either less repulsive or less attractive than the mf result. By studying the individual contributions of the real and imaginary parts of the effective interaction one may gain more insight into this issue (see below). For the two $S$-wave channels the most striking feature of the dressed phase shift is the disappearance of the pairing signature for the ${ }^{1} S_{0}$ channel and the enormous reduction of the signal in the ${ }^{3} S_{1}$ case. While the dressed ${ }^{1} S_{0}$ phase shift is essentially zero at $k_{F}$, it is still clearly attractive at this momentum for the ${ }^{3} S_{1}$ channel. The actual calculation of the phase shift for this channel displays a slight kink close to $k_{F}$, suggesting that the phase shift may actually rise very rapidly to $\pi$ very close to $k_{F}$. This implies a tremendous reduction in the strength of the pairing correlations in this coupled channel as compared to a mf treatment. Gaps of the order of $10 \mathrm{MeV}$ have been obtained for this channel in Refs. [43-45]. Clearly, the dressing of the nucleons has a strong influence on pairing. While one would expect to obtain a gap using dressed nucleons based on the attractive effective interaction at the Fermi surface, its magnitude is presumably drastically reduced as suggested by the phase shift calculation shown in Fig. 10. The main ingredient in this reduction is the decrease in the density of states at $2 \epsilon_{F}$ when dressed nucleons are propagated. As shown in Figs. 3 and 4 this reduction is essentially the square of the strength of the quasiparticle pole at $k_{F}$, leading to a reduction factor of about 0.5 . Since pairing correlations are particularly sensitive to this density of states, it is not surprising that the strength of the pairing is substantially diminished when dressing is taken into account. It is also noteworthy that one observes a smaller negative phase shift for both $S$ waves at higher energy as compared to the $\mathrm{mf}$ 


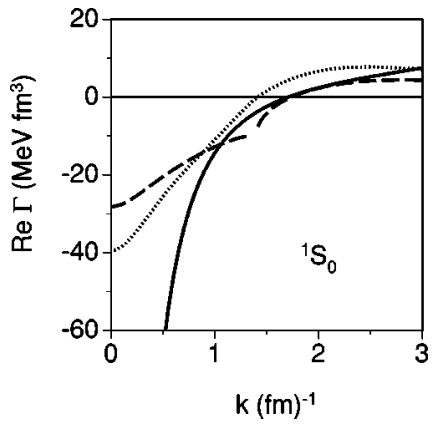

FIG. 11. Real part of the on-shell effective interaction in the ${ }^{1} S_{0}$ channel for free (solid line), $\mathrm{mf}$ (dashed line), and dressed particles (dotted line) as a function of the on-shell momentum.

result. A similar conclusion may be drawn by inspecting the phase shifts for the ${ }^{3} P_{1}$ and ${ }^{3} D_{1}$ channel in the bottom panels of Fig. 10. Also for these partial waves which represent repulsive effective interactions, one observes a reduction of the magnitude of the phase shift when dressing is considered. It is also important to note that in the case of $\mathrm{mf}$ propagation the results in Fig. 10 show that the corresponding results tend to those of free particles at high energy, whereas this is not the case for dressed particles. This latter result indicates that the effect of the dressing extends to a large energy domain. This observation is not too surprising since the spreading of the sp strength due to short-range and tensor correlations takes place in a very large energy domain $[23,24,39]$ and is quite different from a local (in energy) spreading of the strength as would be obtained by a complex quasiparticle energy.

In order to assess the change in the effective interaction when dressing is taken into account, a comparison is made in Figs. 11 and 12 of the real part of the on-shell interaction for free (solid line), $\mathrm{mf}$ (dashed line), and dressed particles (dotted line) for the ${ }^{1} S_{0}$ and ${ }^{3} S_{1}$ interaction, respectively. The free ${ }^{1} S_{0}$ interaction shown in Fig. 11 is quite attractive at low energy reflecting the almost appearance of a bound state. For mf particles this attraction is larger at $k_{F}$ than for free particles at this momentum, leading to bound pair states at this density. Their presence is reflected in the behavior of the real part of the interaction near $k_{F}$ which has been studied in detail in Refs. [43,46]. Using dispersion relations for the real part of the effective interaction one obtains an extremely

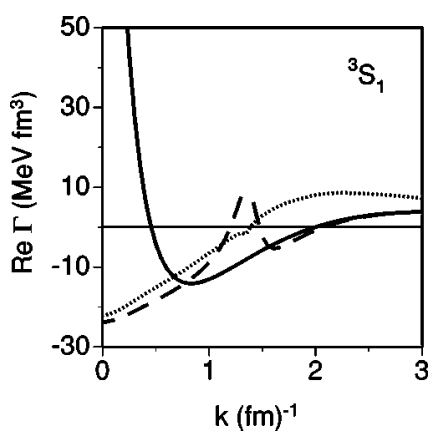

FIG. 12. Real part of the on-shell effective interaction in the ${ }^{3} S_{1}$ channel for free (solid line), $\mathrm{mf}$ (dashed line), and dressed particles (dotted line) as a function of the on-shell momentum.

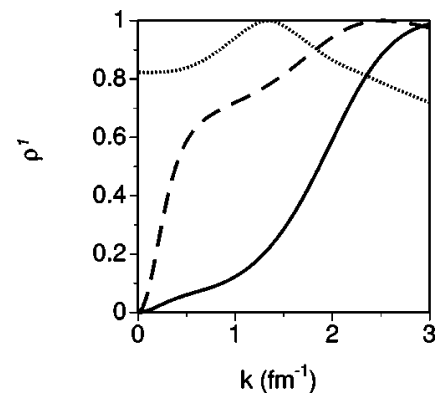

FIG. 13. Mixing parameter for the ${ }^{3} S_{1-}{ }^{3} D_{1}$ channel for free (solid line), $\mathrm{mf}$ (dashed line), and dressed particles (dotted line) as a function of the on-shell momentum.

rapid variation as a function of energy in the case of weakly bound pair states as is the case for the ${ }^{1} S_{0}$ interaction with $\mathrm{mf}$ particles at this density. This behavior cannot be captured as a function of the on-shell momentum on the present scale but is indicated by the kinklike behavior near $k_{F}$. While the effective interaction is less attractive for dressed nucleons at $k_{F}$ than for $\mathrm{mf}$ or free particles, it is more attractive at small momenta (deep in the Fermi sea) than for mf particles. This shows that one may not conclude from a comparison of the phase shifts whether the (real part of the) interaction has actually weakened; quite the opposite is true at small momenta for the ${ }^{1} S_{0}$ channel.

The results for the ${ }^{3} S_{1}$ interaction in Fig. 12 indicate that for free particles the effective interaction becomes very repulsive at low energy. That this should be the case becomes clear when one realizes that the $T$ matrix (effective interaction) has a real pole at the deuteron energy and its real part must therefore approach infinity when the energy approaches the deuteron pole from above. The presence of strongly bound pair states for $\mathrm{mf}$ particles leads to the behavior of the real part of the effective interaction illustrated in Fig. 12 [46]. The dressed effective interaction is weakly attractive at $k_{F}$, suggesting again the substantial weakening of the pairing correlations which are determined by the effective interaction at $k_{F}$. Except near $k_{F}$, the dressed effective interaction is more repulsive than the mf one.

The introduction of a medium with nucleons invites also the question whether the effect of the tensor force can change with respect to its role in free space. Based on the strong pairing in the ${ }^{3} S_{1}$ channel found for $\mathrm{mf}$ particles [23,44,45] one may infer that the tensor force is more effective in the medium than in free space. This can be understood by noting that the lowest energy excitations in the medium have a relative momentum corresponding to $k_{F}$ whereas for free particles the lowest excitation has zero momentum. In the latter case the tensor force cannot directly mix with this low momentum state since the corresponding matrix elements vanish. In order to investigate the role of the tensor force in the medium for $\mathrm{mf}$ and dressed particles, the mixing parameter [Eq. (22)] is displayed in Fig. 13 for free (solid line), mf (dashed line), and dressed particles (dotted line) as a function of the on-shell momentum. Since the eigen mixing parameter is plotted instead of the bar one, the maximum mixing occurs when $\rho^{1}$ equals 1 , corresponding to an equal admixture of the ${ }^{3} S_{1}$ and ${ }^{3} D_{1}$ waves. The "small"' admixture of the ${ }^{3} D_{1}$ 


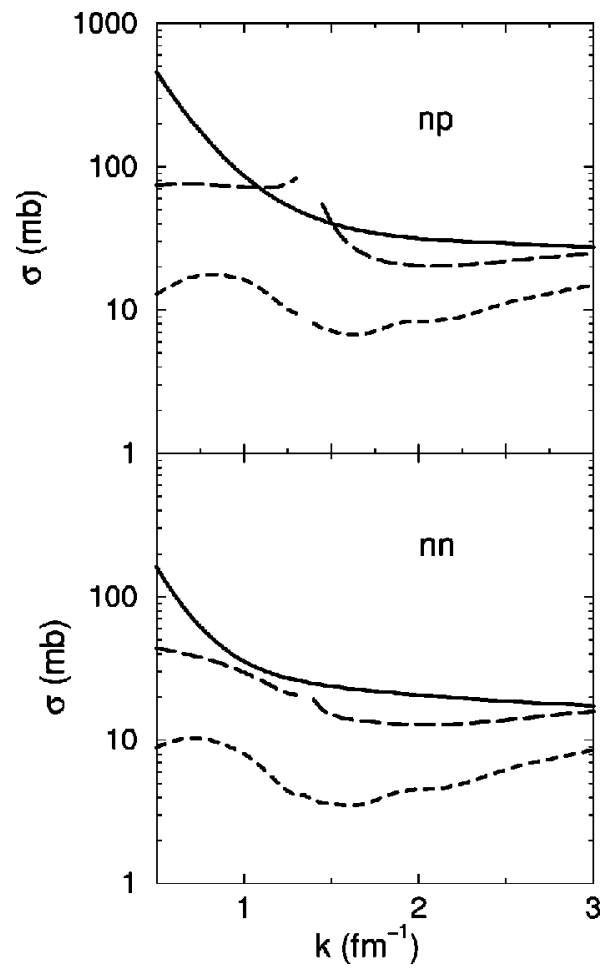

FIG. 14. Total neutron-proton (top) and neutron-neutron (bottom panel) cross sections for free (solid line), mf (dashed line), and dressed particles (short-dashed line) as a function of the on-shell momentum.

wave at small momenta for free particles is apparent and persists for $\mathrm{mf}$ particles. Both for $\mathrm{mf}$ and dressed particles the mixing is substantial over a wide range of momenta. Especially deep in the Fermi sea the mixing parameter is quite large for dressed particles, reaching its maximum around $k_{F}$. It is clear from Fig. 13 that the mixing parameter is quite sensitive to the treatment of the medium and changes its behavior dramatically with respect to free space results.

The results for the phase shifts obtained for dressed particles lead to the expectation that the corresponding total cross sections are substantially reduced compared to the $\mathrm{mf}$ results. The results for neutron-proton $(n p)$ and neutronneutron $(n n)$ total cross sections displayed in Fig. 14 confirm this expectation. Results have been obtained for free (solid line), mf (dashed line), and dressed particles (shortdashed line) by including all partial-wave channels of the Reid potential with $J \leqslant 2$. Results for $\mathrm{mf}$ particles were generated with a realistic sp energy spectrum and are similar to the corresponding results obtained, e.g., in Ref. [17]. The effect of the pairing correlations on the cross sections yields a cusplike behavior around $k_{F}$ reminiscent of the enhancement of the cross sections obtained by the Rostock group at finite temperature $[9,16]$. As the phase shifts for mf particles suggest, the corresponding cross sections in the medium become essentially identical to the one in free space at high energy. Both for the $n p$ and $n n$ total cross sections the effect of dressing the nucleons is quite dramatic, leading to a substantial reduction of the total cross section at all energies. Indeed, on average a cross section of only about $10 \mathrm{mb}$ is obtained. While this may seem a small number, it should be kept in mind that this by no means implies that the effective interaction in the medium has become insignificant (see Figs. 11 and 12). In addition, one should recall that the concept of asymptotic flux in the medium representing preserved information of a scattering event deep in the medium is not a realistic consideration when the dressing of the nucleons is significant [29], as it is at $k_{F}=1.36 \mathrm{fm}^{-1}$. The main ingredient representing the dressing is the two-particle density of states; its reduction for dressed particles is to a large extent responsible for the reduction of the cross section. In this respect it should be noted that the results of Ref. [16] also show a substantial reduction of the total cross section at high energy with increasing temperature. This result implies that with increasing temperature which also means a larger depletion of the Fermi sea due to thermal excitations, one obtains a reduction of the total cross section similar to the one obtained here at zero temperature when the dressing of the particles is incorporated (and therefore the depletion of the Fermi sea due to correlations is included). While no results are shown in Fig. 14 below $0.5 \mathrm{fm}^{-1}$ in order to avoid the large value of the total cross sections for free particles, it should be noted that the cross sections for dressed particles smoothly go to zero when expression (46) is used at lower energies. This expression avoids the problem associated with Eq. (47) which would yield an infinite cross section for the on-shell momentum going to zero. In addition, Eq. (47) does not yield a cross section for energies that do not yield a solution for the on-shell momentum according to Eq. (40), i.e., for energies deep in the hole-hole continuum.

\section{SUMMARY AND CONCLUSIONS}

In the present work results for the scattering of dressed nucleons in the nuclear medium at a density corresponding to $k_{F}=1.36 \mathrm{fm}^{-1}$ have been reported using the Reid soft-core potential. The spectral functions which describe the dressing of the nucleons at this density were taken from Refs. $[39,40]$. These spectral functions describe nucleons with substantial fragmentation of the sp strength, leading to an average occupation number of about 0.8 for momenta below $k_{F}$. The jump in the occupation number at $k_{F}$ embodied in this strength distribution (which also corresponds to the strength of the quasiparticle $z_{k_{F}}$ ) corresponds to 0.72 (see also Ref. [24]). A critical ingredient gauging the difference with the propagation of $\mathrm{mf}$ nucleons is the two-particle density of states. Results for this density of states are shown in Figs. 3 and 4. A substantial reduction for dressed particles is obtained in a wide range of energies with respect to the $\mathrm{mf}$ density of states, the reduction factor being exactly equal to $z_{k_{F}}^{2}$ or about 0.5 at $2 \epsilon_{F}$. For energies below the mf two-hole spectrum a nonvanishing density of states is obtained also on account of the spreading of the sp strength.

While the emphasis in this work is on the results for dressed particles, an attempt has been made to ground the discussion firmly in terms of a comparison of scattering results obtained for mf particles at the same density and at zero temperature. Of particular interest is the connection between pairing correlations and the tendency of the phase shift to 
approach a value of $\pi$ at $2 \epsilon_{F}$. Both the ${ }^{1} S_{0}$ and ${ }^{3} S_{1}{ }^{3} D_{1}$ channels exhibit this behavior at this density when mf propagators are used. Indeed, the phase shift in the coupled channel is quite large and positive over a wide range of energies, suggesting a connection with strong pairing correlations which has been observed for this channel in the literature $[23,44,45]$. The influence of a gap in the sp spectrum or a realistic sp potential energy spectrum was explored for the ${ }^{1} S_{0}$ phase shift. In addition, modifications of the bare ${ }^{1} S_{0}$ interaction were employed to illustrate on the one hand the connection with pairing correlations and a more attractive bare interaction and on the other hand with results for ${ }^{3} \mathrm{He}$ atoms which, also at the $\mathrm{mf}$ level, exhibit a phase shift of $-\pi$ deep in the Fermi sea [31] due to a strongly repulsive core in the interaction. The latter phase shift of $-\pi$ indicates the existence of a bound state below the hole-hole continuum (or at an excitation energy higher than allowed by the removal of two mf particles).

Using expressions proposed in Ref. [29] for the phase shifts and scattering amplitudes for dressed particles, a study has been made of the effect of the dressing of the nucleons on these quantities. The folding procedure in which the effective interaction is sampled with the imaginary part of the dressed (but noninteracting) propagator is shown to yield phase shifts which are quite similar to phase shifts obtained from an "on-shell" prescription for the relative momentum for a wide range of energies. The resulting phase shift for the two nuclear $S$ waves shows that the effect of the dressing is to eliminate the pairing signal for the ${ }^{1} S_{0}$ channel while weakening it substantially for the coupled ${ }^{3} S_{1}-{ }^{3} D_{1}$ channel as shown in Fig. 10. Similar sizable changes are obtained for other partial waves. In all cases one must attribute these changes to the substantial fragmentation of the sp strength as reflected in the substantially different two-particle density of states. While in general both less repulsive and less attractive phase shifts for dressed particles are obtained than for $\mathrm{mf}$ particles, it is not in general true that this always implies a weakening of the effective interaction. An example of this feature is shown in Fig. 11 where the effective ${ }^{1} S_{0}$ interaction inside the Fermi sea is shown to be more attractive for dressed than for mf particles. A particularly sensitive quantity to medium effects is the mixing parameter in the coupled ${ }^{3} S_{1}{ }^{-} D_{1}$ channel. Strong mixing of the ${ }^{3} S_{1}$ and ${ }^{3} D_{1}$ wave functions is obtained for dressed particles in a wide range of energies with a maximum around the Fermi surface.

The results for the two-particle density of states and phase shifts for dressed particles culminate in total cross sections which are substantially smaller than any previously obtained result involving $\mathrm{mf}$ particles at zero temperature. It may be useful to extend the present work to finite temperature to assess the relevance of this reduction for the analysis of heavy-ion reactions. The folding prescription for the total cross section given in Eq. (46) avoids the singularity associated with Eq. (47) at small on-shell momenta. As a result, the total cross sections decrease smoothly for energies going deep into the Fermi sea.

\section{ACKNOWLEDGMENTS}

This work was supported by the National Science Foundation under Grant Nos. PHY-9602127 and PHY-9900713, by DGICYT (Spain) Grant No. PB95-1249, and by Generalitat Catalunya Grant No. 1998SGR-11.
[1] T.T.S. Kuo and G.E. Brown, Nucl. Phys. 85, 40 (1966).

[2] T.T.S. Kuo and G.E. Brown, Nucl. Phys. A114, 241 (1968).

[3] W.H. Dickhoff and H. Müther, Rep. Prog. Phys. 55, 1947 (1992).

[4] C.J.G. Onderwater et al., Phys. Rev. Lett. 78, 4893 (1997).

[5] G. Rosner, in Perspectives in Hadronic Physics, edited by S. Boffi, C. Ciofi degli Atti, and M.M. Giannini (World Scientific, Singapore, 1998), p. 185.

[6] C. Giusti, F.D. Pacati, K. Allaart, W.J.W. Geurts, W.H. Dickhoff, and H. Müther, Phys. Rev. C 57, 1691 (1998).

[7] G.F. Bertsch and S. Das Gupta, Phys. Rep. 160, 189 (1988).

[8] W. Bauer, C.-K. Gelbke, and S. Pratt, Annu. Rev. Nucl. Part. Sci. 42, 77 (1992).

[9] T. Alm, G. Röpke, W. Bauer, F. Daffin, and M. Schmidt, Nucl. Phys. A587, 815 (1995).

[10] B. ter Haar and R. Malfliet, Phys. Rev. C 36, 1611 (1987).

[11] A. Bohnet, N. Ohtsuka, J. Aichelin, R. Linden, and A. Faessler, Nucl. Phys. A494, 349 (1989).

[12] A. Faessler, Nucl. Phys. A495, 103c (1989;).

[13] M. Schmidt, G. Röpke, and H. Schulz, Ann. Phys. (N.Y.) 202, 57 (1990).

[14] G.Q. Li and R. Machleidt, Phys. Rev. C 48, 1702 (1993).

[15] G.Q. Li and R. Machleidt, Phys. Rev. C 49, 566 (1994).

[16] T. Alm, G. Röpke, and M. Schmidt, Phys. Rev. C 50, 31 (1994).
[17] G. Giansiracusa, U. Lombardo, and N. Sandalescu, Phys. Rev. C 53, R1478 (1996).

[18] H.-J. Schulze, A. Schnell, G. Röpke, and U. Lombardo, Phys. Rev. C 55, 3006 (1997).

[19] A. Schnell, G. Röpke, U. Lombardo, and H.-J. Schulze, Phys. Rev. C 57, 806 (1998).

[20] L. Lapikás, Nucl. Phys. A553, 297c (1993).

[21] P. Grabmayr et al., Phys. Lett. 164B, 15 (1985).

[22] G.J. Wagner, in Nuclear Structure at High Spin, Excitation, and Momentum Transfer, edited by Hermann Nann, AIP Conf. Proc. No. 142 (AIP, New York, 1986), p. 220.

[23] B.E. Vonderfecht, W.H. Dickhoff, A. Polls, and A. Ramos, Phys. Rev. C 44, R1265 (1991).

[24] O. Benhar, A. Fabrocini, and S. Fantoni, Nucl. Phys. A550, 201 (1992).

[25] C.C. Gearhart, Ph.D. thesis, Washington University, St. Louis, 1994.

[26] W.H. Dickhoff, C.C. Gearhart, J. Yuan, A. Polls, and A. Ramos, in Condensed Matter Theories, edited by M. Casas, M. de Llano, J. Navarro, and A. Polls (Nova Science, Commack, NY, 1995), Vol. 10, p. 349.

[27] W.H. Dickhoff et al., in Perspectives in Nuclear Physics at Intermediate Energies, edited by S. Boffi, C. Ciofi degli Atti, and M.M. Giannini (World Scientific, Singapore, 1996), p. 296. 
[28] W.H. Dickhoff, in Recent Progress in Many-Body Theories, edited by D. Neilson and R.F. Bishop (World Scientific, Singapore, 1998), p. 216.

[29] W.H. Dickhoff, Phys. Rev. C 58, 2807 (1998).

[30] R.V. Reid, Ann. Phys. (N.Y.) 50, 411 (1968).

[31] R.F. Bishop, H.B. Ghassib, and M.R. Strayer, Phys. Rev. A 13, 1570 (1974).

[32] K. Gottfried, Quantum Mechanics (Addison-Wesley, Redwood City, CA, 1989).

[33] E. Roth, W.H. Dickhoff, A. Polls, and A. Ramos (unpublished).

[34] J.M. Blatt and L.C. Biedenharn, Phys. Rev. 86, 399 (1952).

[35] G.E. Brown and A.D. Jackson, The Nucleon-nucleon Interaction (North-Holland, Amsterdam, 1976).

[36] M.I. Haftel and F. Tabakin, Nucl. Phys. A158, 1 (1970).

[37] F.A. Brieva and J.R. Rook, Nucl. Phys. A291, 299 (1977).

[38] H.R. Glyde and S.I. Hernadi, Phys. Rev. B 28, 141 (1983).
[39] B.E. Vonderfecht, W.H. Dickhoff, A. Polls, and A. Ramos, Nucl. Phys. A555, 1 (1993).

[40] C.C. Gearhart, W.H. Dickhoff, A. Polls, and A. Ramos, Int. J. Mod. Phys. E 5, 261 (1996).

[41] M. Trefz, A. Faessler, and W.H. Dickhoff, Nucl. Phys. A443, 499 (1985).

[42] J.M. Chen, J.W. Clark, E. Krotscheck, and R.A. Smith, Nucl. Phys. A451, 509 (1986).

[43] B.E. Vonderfecht, C.C. Gearhart, W.H. Dickhoff, A. Polls, and A. Ramos, Phys. Lett. B 253, 1 (1991).

[44] T. Alm, G. Röpke, and M. Schmidt, Z. Phys. A 337, 355 (1990).

[45] M. Baldo, I. Bombaci, and U. Lombardo, Phys. Lett. B 283, 8 (1992).

[46] B.E. Vonderfecht, Ph.D. thesis, Washington University, St. Louis, 1991. 